\title{
Diagnóstico energético del sistema de calentamiento de agua en la piscina del Complejo de Natación Carlos Costales de la ciudad de Riobamba. Propuesta de alternativas de solución
}

Energy diagnosis of the water heating system in the swimming pool of the Carlos Costales Swimming Complex in the city of Riobamba. Proposal of alternative solutions

Ligia Elena Moreno Pinduisaca ${ }^{1}$ Juan Carlos Palacios Almache ${ }^{2}$ Luis Fernando Buenaño Moyano ${ }^{3}$ Olga Beatriz Barrera Cárdenas ${ }^{4}$

Recibido: 13-03-2019 / Revisado: 25-04-2019 /Aceptado: 24-05-2019/ Publicado: 05-06-2019

\begin{abstract}
DOI: https://doi.org/10.33262/cienciadigital.v3i2.6.572

In the swimming pool of the "Carlos Costales" swimming complex, an energy diagnosis of the water heating system using a diesel boiler for its operation is needed. The heat losses calculated are $84.573 \mathrm{KW}$ on average, the heat delivered by the boiler to heat the pool water from $06 \mathrm{H} 00$ to $10 \mathrm{H} 30$ is $103.700 \mathrm{KW}$ and from $10 \mathrm{H} 30$ to $19 \mathrm{H} 00$ is $94.016 \mathrm{KW}$, performance of the boiler $58.9 \%$ and $53.4 \%$ respectively, a year with this system produced 131206 tons of $\mathrm{CO} 2$. The average fuel consumption is 3.2 gals / h, which represents an expense for the State of \$21,027.79 per year. To meet the needs of bathers with a temperature of $28{ }^{\circ} \mathrm{C}$ ideal for training children from 6 years old, the use of a hybrid system with four hours of operation of the boiler and 93 solar panels type $\mathrm{HC}$ - 50, an annual fuel savings of $\$ 14,797.77$ is generated and the calculated IRR for this option is $19.4 \%$, which is why it is considered a feasible project, emission of polluting gases from 131.20 to 38.87 tons of $\mathrm{CO} 2$, which represent approximately $70 \%$ less environmental pollution.
\end{abstract}

Key Words: Diagnosis, Hybrid, Fuel, Solar, Energy.

\footnotetext{
${ }^{1}$ Escuela Superior Politécnica de Chimborazo, Chimborazo, Ecuador, ligiam4183@ hotmail.es

${ }^{2}$ Petroamazonas EP, Chimborazo, Ecuador, juank2981@ hotmail.com

${ }^{3}$ Escuela Superior Politécnica de Chimborazo, Chimborazo, Ecuador, lfbuenanio@espoch.edu.ec

${ }^{4}$ Escuela Superior Politécnica de Chimborazo, Chimborazo, Ecuador, obarrera@espoch.edu.ec
} 


\section{Resumen}

En la Piscina del complejo "Carlos Costales" se realizó un diagnóstico energético del sistema de calentamiento de agua que utiliza una caldera a diesel. Las pérdidas de calor calculadas son de $84,573 \mathrm{~kW}$ en promedio, para calentar el agua de la piscina de $6 \mathrm{H} 00 \mathrm{a}$ $10 \mathrm{H} 30$ la caldera entrega $103,700 \mathrm{~kW}$ y de $10 \mathrm{H} 30$ a $19 \mathrm{H} 0094,016 \mathrm{~kW}$, con un rendimiento de la caldera de 58,9\% y 53,4\% respectivamente, al año con este sistema se producen 131 206 toneladas de CO2. El consumo promedio de combustible es de 3,2 gal/h lo que representa un gasto para el Estado de $\$ 21.027,79$ al año. Para satisfacer las necesidades de los bañistas con una temperatura de $28^{\circ} \mathrm{C}$ ideal para el entrenamiento de niños desde los 6 años de edad se plantea un sistema híbrido con cuatro horas de funcionamiento de la caldera y 93 paneles solares tipo HC - 50, anualmente se generará un ahorro en el gasto de combustible de $\$ 14.797,77$ y el TIR calculado para esta opción es de 19,4\% que se considera un proyecto factible, se reducirá al año la emisión de 131,20 a 38,87 toneladas de CO2 que representa el $70 \%$ menos de contaminación ambiental.

Palabras Clave: Diagnóstico, Híbrido, Combustible, Solares, Energético.

\section{Introducción}

En la actualidad la energía en forma de calor es un recurso ampliamente utilizado, sin embargo la utilización de energías renovables y limpias en varios lugares es bastante limitado lo que representa un alto consumo de combustibles fósiles y por lo tanto un importante costo medioambiental.

El uso ineficiente de la energía es un problema a nivel mundial porque utiliza cantidades excesivas de los escasos recursos no renovables, al incrementar la eficiencia de la energía, se reduce el uso de recursos de energía, por lo que de este modo se retarda su agotamiento, se disminuye los impactos negativos sobre el medio ambiente y la salud. En el continente Americano varios países como Cuba, Nicaragua, Chile, Perú y Brasil han apostado por generar estrategias que les permita utilizar energías limpias y dejar la dependencia de los hidrocarburos y proyectos carboníferos, ya que el actual modelo energético es insostenible, lo que conlleva necesariamente a pensar en un futuro energético combinado racionalmente para hogares, piscinas, hospitales, hoteles y procesos industriales, (Linkohr, 2006).

En el Ecuador se ha optado por una política que incurre en la utilización de fuentes de energía limpia y renovable de tal forma que se minimice la contaminación ambiental y se fomente el desarrollo y la implementación de equipos que utilicen energía producida dentro del país como la eléctrica.

Calentar el agua de una piscina significa un uso de energía muy alto por lo que una producción más limpia en este proceso significa una de las aplicaciones más lógicas, aunque en cuestión de números representa una inversión inicial mayor comparada con los sistemas instalados convencionalmente como es el calentamiento con derivados de petróleo. En la ciudad de Riobamba actualmente se utiliza los sistemas convencionales de energía en el calentamiento de agua de las 
piscinas existentes, en donde no se ejerce un control adecuado de la contaminación producida por la utilización de hidrocarburos.

La piscina del Complejo de Natación "Carlos Costales", es semiolímpica, el agua se calienta a través de un calentador a diésel, la falta de ingresos económicos y el alto costo de mantenimiento hicieron que el complejo cierre las instalaciones por algún tiempo, actualmente la piscina se encuentra en funcionamiento de martes a domingo en un horario ininterrumpido desde las $07 \mathrm{H} 00$ hasta las 20H00, es utilizada para la formación de niños, jóvenes y adultos de la provincia, en lo que conlleva a la disciplina deportiva de natación y su administración está a cargo de la Federación Deportiva de Chimborazo.

A través de una inspección se identificó que la temperatura alcanzada no es la adecuada ya que no se tiene un abastecimiento oportuno de combustible, esto provoca que en algunas ocasiones no se pueda calentar el agua de la piscina por varios días lo que genera malestar en los usuarios. Si no se corrige el problema del sistema de calentamiento de agua se corre el riesgo de reducir el número de usuarios afectando así los ingresos económicos y el desarrollo deportivo de la provincia en la disciplina de natación, además de una eminente contaminación ambiental.

Una posible solución para alcanzar la temperatura adecuada del agua de la piscina es la utilización de un sistema de calentamiento combinado, ya sea con la utilización de energía eléctrica o paneles solares, lo que reducirá el uso de hidrocarburos y la contaminación ambiental producida al calentar agua.

\section{Importancia del Problema}

El aporte de este estudio permitirá obtener un menor costo y mayor eficiencia en la operación de la piscina, favoreciendo de esta manera a la Federación Deportiva de Chimborazo que está a cargo de la misma, como a los usuarios que en su gran mayoría son deportistas los mismos que se forman para distintas competencias nacionales, para estos se cuenta con el apoyo de la FDCH que está de acuerdo con el desarrollo investigativo y prestará todas las facilidades para que se pueda tomar datos y mediciones, los cuales permitan conocer la realidad del funcionamiento de la piscina, con el fin de establecer los correctivos necesarios y además en un futuro de acuerdo a los resultados económicos pensar en la implementación de un sistema de calentamiento de agua combinado y de alta eficiencia.

\section{Metodología}

El estudio para este proyecto se basa en seleccionar una alternativa que pueda reemplazar o ayudar a la caldera que está instalada para lograr suministrar energía al agua de la piscina y que tenga una temperatura óptima, estos parámetros pueden ser determinados a través de una investigación de campo y mediante el uso de variables cuantificables que permitan desarrollar un planteamiento matemático para demostrar las suposiciones planteadas. 
Para el desarrollo del módulo descriptivo y la elaboración de los cálculos es adecuado llegar una investigación bibliográfica, que permita insertar referencias, tomar ecuaciones, datos, técnicas y métodos que faciliten dichos cálculos.

\section{- Tipo de investigación}

Se realizará una investigación descriptiva donde se buscará especificar los parámetros necesarios para lograr la eficiencia energética en el complejo, se establecerá diversos aspectos como dimensiones o componentes del sistema de calentamiento actual, se estudiará e interpretará sistemas de calentamiento que funcionen con energías alternativas.

Otro tipo de investigación que se realizará es de tipo explicativo se dará a conocer la temperatura óptima, la cantidad de combustible que se utilizará en el caldero si se instala un sistema secundario de apoyo teniendo en cuenta los beneficios que se puede brindar a la población y minimizando la contaminación ambiental.

\section{- Técnicas e instrumentación de la investigación}

Las técnicas e instrumentos que se utilizarán para determinar las características específicas de cada variable de acuerdo a los objetivos específicos planteados son determinadas en la tabla 1.

Tabla 1: Técnicas e instrumentos de la investigación

\begin{tabular}{ll}
\hline Objetivos específicos & $\begin{array}{l}\text { Métodos y Técnicas de } \\
\text { investigación }\end{array}$ \\
\hline $\begin{array}{l}\text { Desarrollar una auditoria energética } \\
\text { preliminar. }\end{array}$ & - Observación - registro \\
Determinar la cantidad de calor necesario & - Medición \\
para alcanzar la temperatura adecuada & - Medición \\
del agua en la piscina. & - Cálculos - ecuaciones \\
$\begin{array}{l}\text { Plantear un sistema de calentamiento de } \\
\text { agua eficiente y que utilice energías } \\
\text { alternativas. }\end{array}$ & - Revisión bibliográfica \\
$\begin{array}{l}\text { Realizar un estudio de impactos de la la ecuaciones } \\
\text { propuesta. }\end{array}$ & Cálculos - ecuaciones \\
\hline
\end{tabular}

Fuente: Autor, Ecuador, 2018

En la tabla 2 se detalla la variable física que se va a medir, qué instrumento se utilizará para su medición, el parámetro medido y para qué cálculo se utilizarán. 
Tabla 2: Variable física

\begin{tabular}{|c|c|c|c|}
\hline Variable física & $\begin{array}{l}\text { Instrumento } \\
\text { medida }\end{array}$ & Parámetro medido & Tipo de cálculo \\
\hline Longitud & Flexómetro & $\begin{array}{l}\text { Longitud de la } \\
\text { piscina }\end{array}$ & Volumen \\
\hline Temperaturas & Termómetro digital & $\begin{array}{l}\text { - Agua de la piscina } \\
\text { - Agua de la red } \\
\text { - Agua de reposición }\end{array}$ & $\begin{array}{l}\text { Pérdidas de calor } \\
\text { Eficiencia de la } \\
\text { caldera. }\end{array}$ \\
\hline Temperatura & Termo higrómetro & Temperatura del aire & Pérdidas de calor \\
\hline Temperatura & Pirómetro & $\begin{array}{l}\text { Temperatura de las } \\
\text { paredes de la piscina }\end{array}$ & Pérdidas de calor \\
\hline $\begin{array}{l}\text { Humedad } \\
\text { relativa }\end{array}$ & Termo higrómetro & Humedad del aire & Pérdidas de calor \\
\hline
\end{tabular}

Fuente: Autor, Ecuador, 2018.

\section{Resultados y Discusión}

\section{Funcionamiento del sistema de calentamiento de agua}

Las etapas que forman parte del proceso de calentamiento de agua son:

\section{Succión del agua de la piscina}

El proceso de calentamiento del agua empieza en la succión por medio de seis skimmers que se encuentran ubicados en la piscina tres en cada lado a lo largo de la misma, para posteriormente por medio de gravedad y con la ayuda de un sistema de tuberías de diámetro de 3 pulgadas llegar hasta la sala de máquinas, donde se inicia el proceso de calentamiento y recirculación del agua.

\section{Proceso de calentamiento de agua}

Una vez que por medio de la tubería de succión el agua llega a la sala de máquinas, esta se encuentra con un juego de válvulas donde mediante la apertura o cierre se elige hacia cual bomba hidráulica se destinará el flujo.

El sistema de recirculación del agua cuenta con dos bombas que funcionan alternadamente según el plan de operación del sistema, la cual proporciona energía al fluido para que este circule a través del sistema de calentamiento de agua y posteriormente sea descargada nuevamente a la piscina.

De las dos bombas existentes en el sistema siempre está en funcionamiento solo una, ya que es suficiente para proveer de la energía necesaria al fluido que circula por el sistema de tuberías. El agua succionada después de pasar por la bomba ingresa a los filtros los cuales se encargan de eliminar las impurezas o residuos que puedan existir en el sistema, para que no afecten el funcionamiento de la caldera. 


\section{Descarga del agua hacia la piscina}

Cuando el fluido gana temperatura al momento de circular por la caldera es inyectada nuevamente a la piscina por medio del sistema de tuberías de descarga y 14 impulsores situados uniformemente en todo el contorno de la piscina.

En la figura 1 se representa gráficamente el sistema de calentamiento de agua de la piscina "Carlos Costales".

Figura 1: Sistema de calentamiento de agua de la piscina "Carlos Costales"

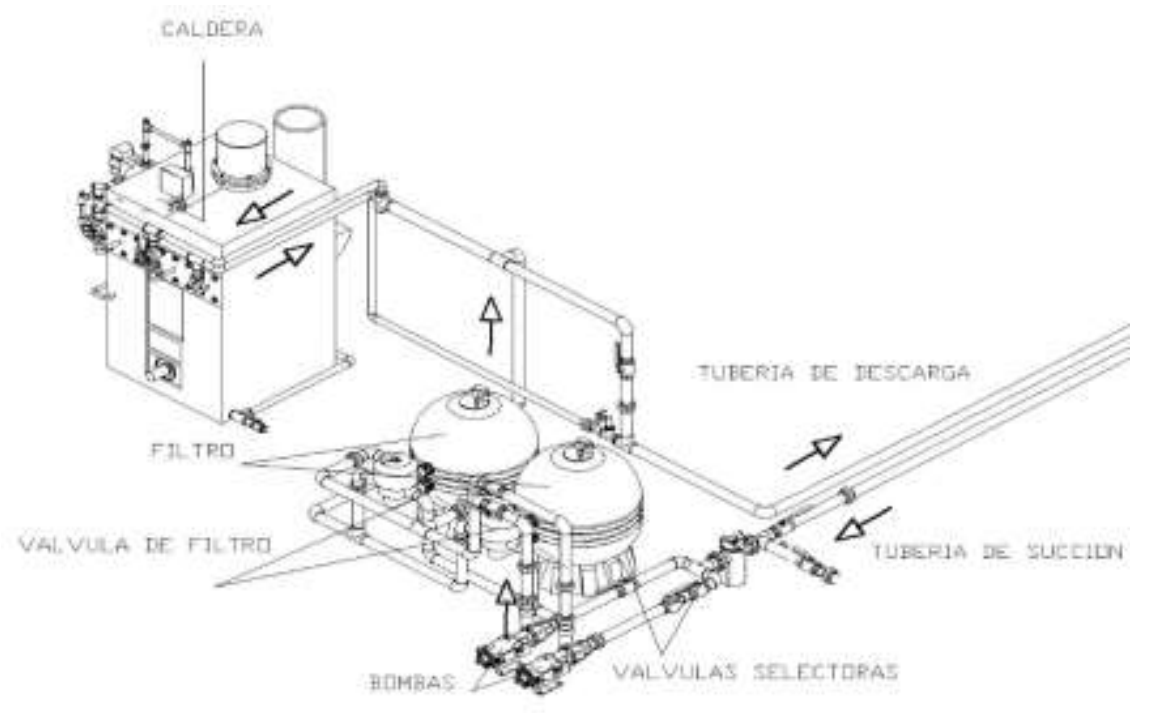

Fuente: Autor, Ecuador, 2018

\section{Toma de datos y registro}

Utilizando el termómetro digital con sonda intercambiable PT 100 se midió la temperatura del agua de la piscina antes de encender la caldera y cuando está encendida, así como del agua de reposición y se muestra en la tabla 3:

Tabla 3: Temperaturas promedio de agua

\begin{tabular}{ll}
\hline DATOS PROMEDIO DE TEMPERATURA & ${ }^{\circ} \mathbf{C}$ \\
\hline Temperatura de agua de reposición & 16,0 \\
Temperatura del agua de la piscina a las $6 \mathrm{H} 00$ & 24,4 \\
Temperatura de agua de la piscina & 26,65 \\
\hline
\end{tabular}

Fuente: Autor, Ecuador, 2018

Según los datos registrados en la tabla 3 se concluye que la temperatura del agua que alcanza la piscina está dentro del rango de temperaturas adecuadas para entrenamiento de natación que según (Ciatesa, 2005) es de $26^{\circ} \mathrm{C}$, sin embargo hay que considerar que a la piscina asisten niños y la temperatura del agua de una piscina infantil es de $30^{\circ} \mathrm{C}$. 


\section{Pérdidas de calor en la piscina}

Los datos obtenidos en las instalaciones de la piscina son los que se muestran en la tabla 4:

Tabla 4: Datos utilizados en el cálculo de las pérdidas de calor

\begin{tabular}{ll}
\hline Temperatura del agua & $26,65^{\circ} \mathrm{C}$ \\
\hline Temperatura ambiente & $22,15^{\circ} \mathrm{C}$ \\
Humedad & \multicolumn{2}{c}{$86,95 \%$} \\
$\begin{array}{l}\text { Número de ocupantes de } \\
\text { piscina }\end{array}$ & 28 \\
Número de espectadores & 6 \\
Superficie de la piscina & $312,87 \mathrm{~m}^{2}$ \\
Volumen de la piscina & $746,26 \mathrm{~m}^{3}$ \\
\hline
\end{tabular}

Fuente: Autor, Ecuador, 2018

Las dimensiones corresponden a una piscina semiolímpica cuya profundidad varía, la humedad relativa está por encima del valor permitido, de acuerdo a (cosasdepiscina.wordpress.com, 2014) indica que la humedad relativa del aire de las piscinas cubiertas no excederá del 65\% para proteger los cerramientos de la formación de condensaciones, sin embargo se acepta como límite el $80 \%$ y con respecto a la temperatura del aire estará entre $2^{\circ} \mathrm{C}$ y $4^{\circ} \mathrm{C}$ por encima que la del agua, esto no ocurre en el presente caso de estudio.

\section{Resumen de pérdidas de calor $\left(Q_{R}\right)$ en la piscina}

En la tabla 5 se presenta un resumen de las pérdidas de calor en la piscina en operación y en reposo:

Tabla 5: Resumen de pérdidas de calor en la piscina

\begin{tabular}{|c|c|c|c|c|}
\hline \multicolumn{5}{|c|}{ PÉRDIDAS DE CALOR EN LA PISCINA } \\
\hline \multirow[b]{2}{*}{ P. EVAPORACION } & $\begin{array}{ll}\mathbf{E N} \quad \mathbf{O} \\
(\mathbf{W})\end{array}$ & OPERACIÓN & \multicolumn{2}{|c|}{ EN REPOSO (W) } \\
\hline & 39024,79 & 39,024 & 22123,63 & 22,124 \\
\hline P. RADIACION & 12149,35 & 12,149 & 12149,35 & 12,149 \\
\hline P. CONVECCION & 1362,20 & 13,622 & 1362,20 & 1,362 \\
\hline P. RENOVACION & 28896,13 & 28,896 & 0 & 0 \\
\hline P. CONDUCCION & 3141,31 & 3,141 & 3141,31 & 3,141 \\
\hline TOTAL & 84573,78 & 84,573 & 38776,49 & 38,776 \\
\hline
\end{tabular}

Fuente: Autor, Ecuador, 2018

Las pérdidas de calor se calcularon cuando el sistema estaba en funcionamiento durante 13 horas y también cuando el sistema se encontraba en reposo tomando en cuenta que durante este tiempo no hay nadadores por lo que las pérdidas por evaporación disminuyen y las pérdidas por renovación desaparecen porque no hay reposición de agua. 
La tabla 6 registra las pérdidas de calor en la piscina en diferentes condiciones de funcionamiento y a diferentes horas, lo que significa que varía también el número de ocupantes de la piscina por ende las pérdidas de calor en operación cambian de valor.

Tabla 6: Resumen de pérdidas de calor en la piscina en diferentes condiciones de funcionamiento

\begin{tabular}{llll}
\hline T. agua piscina & $26,00^{\circ} \mathrm{C}$ & $26,65{ }^{\circ} \mathrm{C}$ & $26,44{ }^{\circ} \mathrm{C}$ \\
\hline Hora & $9 \mathrm{H} 00$ & $11 \mathrm{H} 00$ & $15 \mathrm{H} 00$ \\
\# nadadores & 20 & 28 & 37 \\
\# espectadores & 4 & 6 & 6 \\
P. Evaporación & 34182,49 & 39024,79 & 44319,91 \\
P. Radiación & 11353,54 & 12149,35 & 12149,35 \\
P. Convección & 1179,18 & 1362,20 & 1362,20 \\
P. Renovación & 27678,28 & 28896,12 & 28896,12 \\
P. Conducción & 2819,12 & 3141,31 & 3141,31 \\
TOTAL $(\mathbf{W})$ & $\mathbf{7 7 ~ 2 1 2 , 6 1}$ & $\mathbf{8 4 ~ 5 7 3 , 7 7}$ & $\mathbf{8 9 8 6 8 , 8 9}$ \\
\hline
\end{tabular}

Fuente: Autor, Ecuador, 2018

En la figura 1 se observa la variación del número de ocupantes de la piscina en el eje horizontal, mientras este número aumenta las pérdidas de calor en operación también lo hacen de acuerdo al eje vertical, esto se debe a que las pérdidas de calor por evaporación dependen específicamente del número de ocupantes de la piscina.

Figura 1: Análisis de las pérdidas de calor para diferentes condiciones de funcionamiento

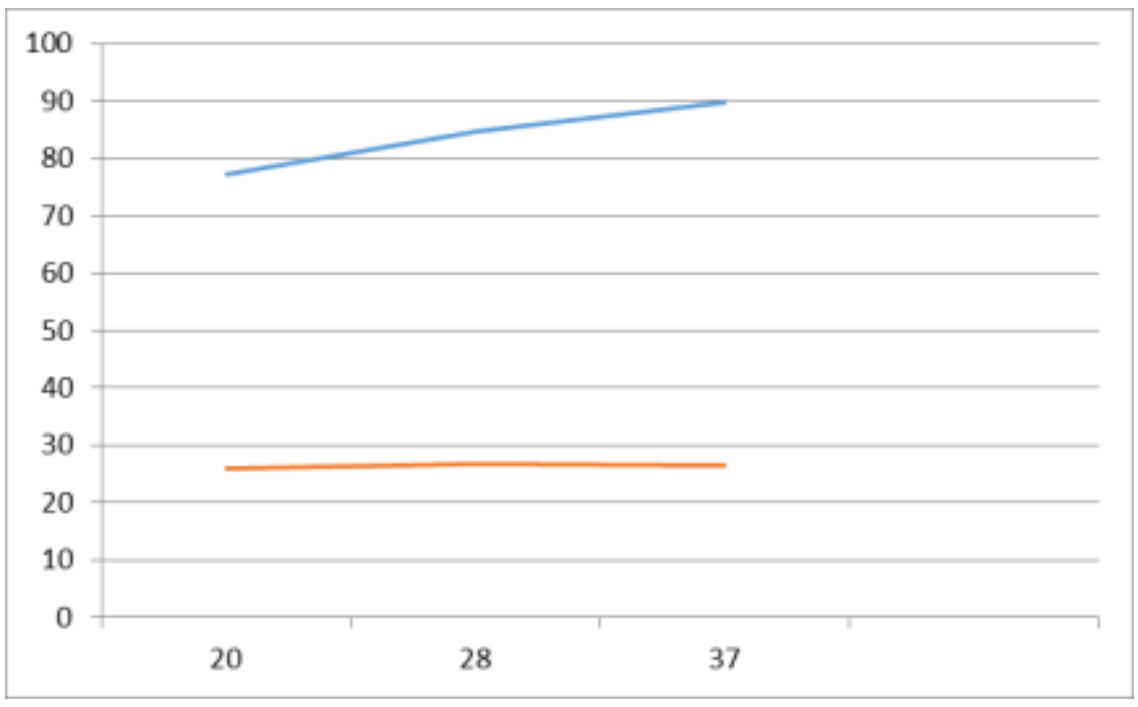

Fuente: Autor, Ecuador, 2018 


\section{Cálculo del calor necesario para calentar el agua de la piscina}

Se tomó en cuenta dos periodos de operación de la caldera el uno está considerado desde las $6 \mathrm{H} 00$, en el que, la temperatura del agua de la piscina inicia a $24,4^{\circ} \mathrm{C}$ hasta las $10 \mathrm{~h} 30$, en donde la temperatura del agua alcanza $26,65^{\circ} \mathrm{C}$, y el segundo periodo se calcula para las horas restantes de operación a partir de las 10H30 tiempo en el cual es prendida la bomba para que recircule el agua de la piscina hasta las $19 \mathrm{H} 00$ y se requiere solamente mantener la temperatura del agua, tabla 7 :

Tabla 7: Calor necesario del agua de la piscina

\begin{tabular}{|c|c|c|}
\hline $\boldsymbol{P}_{\mathbf{0}}$ & Periodo 1 (W) & Periodo 2 (W) \\
\hline$Q$ & $6,84 \frac{\mathrm{m}^{3}}{\mathrm{~h}}$ & $6,84 \frac{\mathrm{m}^{3}}{\mathrm{~h}}$ \\
\hline $\mathrm{D}$ & $998,47 \frac{\mathrm{Kg}}{\mathrm{m}^{3}}$ & $998,47 \frac{\mathrm{Kg}}{\mathrm{m}^{3}}$ \\
\hline$C_{e}$ & $1,1680 \frac{\mathrm{Wh}}{\mathrm{Kg}^{\circ} \mathrm{C}}$ & $1,1618 \frac{\mathrm{Wh}}{\mathrm{Kg}^{\circ} \mathrm{C}}$ \\
\hline$T_{C C}$ & $92^{\circ} \mathrm{C}$ & $38,50{ }^{\circ} \mathrm{C}$ \\
\hline$T_{f C}$ & $79{ }^{\circ} \mathrm{C}$ & $26,65{ }^{\circ} \mathrm{C}$ \\
\hline$P_{0}$ & $103699,656 \mathrm{~W}$ & $94016,366 \mathrm{~W}$ \\
\hline
\end{tabular}

Fuente: Autor, Ecuador, 2018

Se concluye que el calor entregado por la caldera para calentar el agua de la piscina en el periodo 1 es de $103,700 \mathrm{~kW}$ y para el periodo dos es de $94,016 \mathrm{~kW}$, estos valores sirven para calcular el rendimiento de la caldera.

\section{Calor entregado por el quemador a la caldera}

Para determinar el calor entregado por el quemador se consideraron las especificaciones técnicas del equipo que se detallan a continuación en la tabla 8:

Tabla 8: Calor entregado por el quemador

\begin{tabular}{lll}
\hline \multicolumn{3}{l}{ Especificaciones del quemador } \\
\hline 83 & $\mathrm{~kW}$ & Potencia mínima \\
176 & $\mathrm{~kW}$ & Potencia máxima \\
7 & $\mathrm{~kg} / \mathrm{h}$ & Flujo de combustible mínimo \\
14,8 & $\mathrm{~kg} / \mathrm{h}$ & $\begin{array}{l}\text { Flujo de combustible } \\
\text { máximo }\end{array}$ \\
\hline
\end{tabular}

Fuente: Autor, Ecuador, 2018 
Para calentar el agua de la piscina a la temperatura adecuada en el menor tiempo posible, el quemador está regulado para consumir el máximo del flujo de combustible y tomando en cuenta que el equipo es nuevo se considera q entrega el máximo de la potencia al sistema

\section{Rendimiento de la caldera}

Se determinó el rendimiento de la caldera para los dos períodos de funcionamiento, sus valores se muestran en la tabla 9:

Tabla 9: Rendimiento de la caldera

\begin{tabular}{lll}
\hline \multicolumn{2}{l}{ Periodo 1 } & Periodo 2 \\
\hline $\mathrm{P}_{0}$ & $103699,656 \mathrm{~W}$ & $94016,366 \mathrm{~W}$ \\
$\mathrm{P}_{\mathrm{g}}$ & $176 \mathrm{~kW}$ & $176 \mathrm{~kW}$ \\
$\mathrm{n}$ & $58,9 \%$ & $53,4 \%$ \\
\hline
\end{tabular}

Fuente: Autor, Ecuador, 2018

Se obtiene dos valores para el rendimiento de la caldera, el primer valor de 58,9\% corresponde al periodo $1 \mathrm{y}$ el valor de $53,4 \%$ al periodo 2 , como se lo muestra en la siguiente figura 2

Figura 2: Análisis de la eficiencia de la caldera

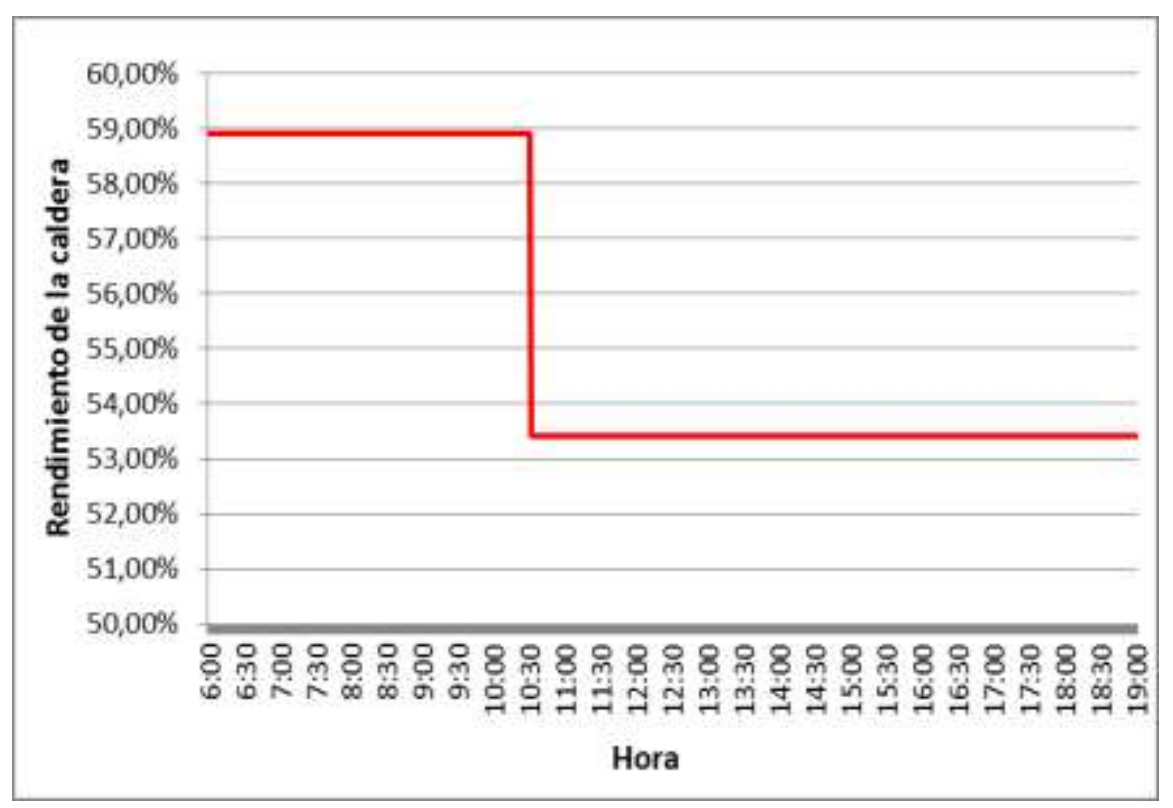

Fuente: Autor, Ecuador, 2018

En la gráfica 3.2 se observa la variación de la eficiencia de la caldera en relación a los dos periodos mencionados anteriormente, de $6 \mathrm{H} 00$ a $10 \mathrm{H} 30$ la eficiencia es de $58,92 \%$, en el segundo periodo de $10 \mathrm{H} 30$ a $19 \mathrm{H} 00$ la eficiencia baja a 53,42 ya que el quemador se prende cada hora durante 30 minutos y se apaga. 


\section{Consumo del combustible}

El consumo de combustible difiere en dos etapas la primera desde las 5H30, hora en la que se enciende el quemador y la temperatura del agua en el interior de la piscina es de $24,4^{\circ} \mathrm{C}$ hasta las $10 \mathrm{H} 30$, en esta etapa el quemador se encuentra en operación continua con un flujo de combustible máximo de $14,8 \mathrm{~kg} / \mathrm{h}$ y la segunda etapa a partir de las $10 \mathrm{H} 30$ en la que el quemador actúa en una operación intermitente, se prende y se apaga cada hora durante 30 minutos, ya que solo se necesita mantener estable la temperatura del agua en la piscina como se presenta en la figura 3 :

Figura 3: Consumo de combustible

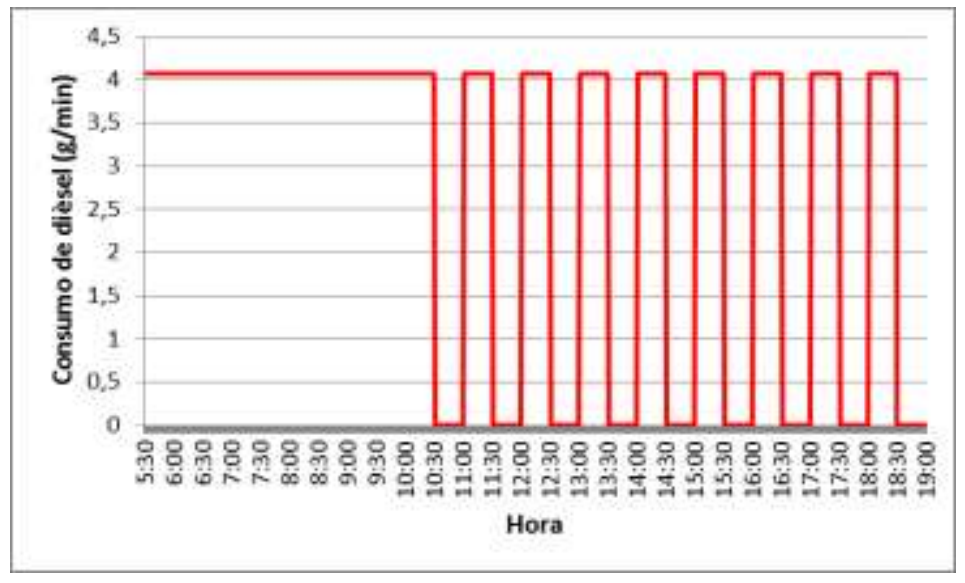

Fuente: Autor, Ecuador, 2018

La temperatura del agua de la piscina también varía durante el tiempo de funcionamiento, la temperatura es de $24,4{ }^{\circ} \mathrm{C}$, la misma que aumenta de valor a $26,65^{\circ} \mathrm{C}$ en las primeras 5 horas de operación del sistema, en las siguientes horas la temperatura permanece aproximadamente constante como se observa en la figura 4, esta etapa corresponde al encendido y apagado intermitente del quemador.

Figura 4: Temperatura del agua de la piscina

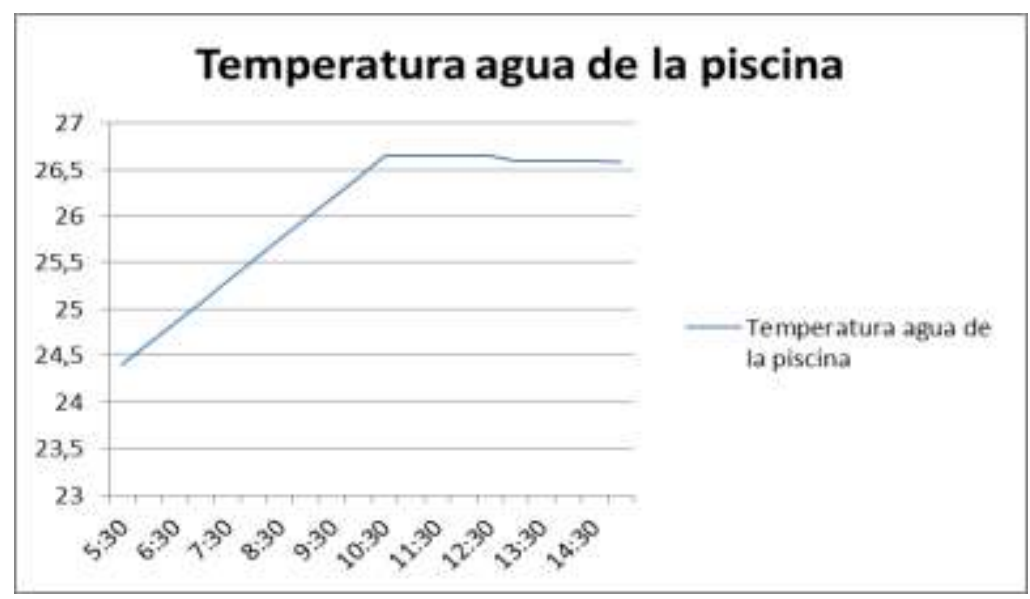

Fuente: Autor, Ecuador, 2018 


\section{Análisis del consumo de diesel}

El consumo de diesel se determina en relación al flujo de combustible y su densidad el consumo máximo de combustible según especificaciones del quemador es de 4,6706 gal/h.

El tiempo que el quemador está encendido es de 9,25 horas (periodo $1+$ periodo 2), entonces para calentar el agua de la piscina se utiliza 43,20 galones, para el consumo promedio de combustible se utiliza las 13,5 horas de funcionamiento del sistema lo que da como resultado $3,2 \mathrm{gal} / \mathrm{h}$ por lo que al mes son necesarios 1 036,87 galones de diesel.

\section{Costo del combustible utilizado en el funcionamiento del sistema}

El costo de operación del sistema se calcula en dos escenarios, el costo de diesel con subsidio del Estado y el costo sin subsidio que representa el valor real del costo de operación. El galón de diesel según (globalpetrolprices.com, 2018) es de 1,03 dólares con subsidio y de 1,69 dólares sin subsidio, estos datos se registran en la tabla 10.

Tabla 10: Costo del combustible utilizado en el sistema

\begin{tabular}{lcll}
\hline Consumo & & $\begin{array}{l}\text { Costo diesel /gal } \\
\mathbf{1 , 0 3} \text { \$ Subsidio }\end{array}$ & $\begin{array}{l}\text { Costo diesel /gal } \\
\mathbf{1 , 6 9} \text { \$ Sin Subsidio }\end{array}$ \\
\hline 4,6707 & $\mathrm{gal} / \mathrm{h}$ & 4,81 & 7,89 \\
1036,87 & $\mathrm{gal} / \mathrm{mes}$ & 1067,98 & 1752,31 \\
12442,48 & $\mathrm{gal} / \mathrm{año}$ & 12815,75 & 21027,79 \\
\hline
\end{tabular}

Fuente: Autor, Ecuador, 2018

De acuerdo a los precios de diesel en el Ecuador el costo de combustible para calentar el agua de la piscina es de $\$ 1067,98$ al mes, al año corresponde a $\$ 12815,75$ siempre y cuando se maneje el subsidio del combustible, el costo aumenta si se comprase el diesel sin subsidio, al mes se tiene un gasto de $\$ 1752,31$ aumentando su valor alrededor de 700 dólares, lo que representa al año $\$ 8$ 400 más.

\section{Eficiencia de la combustión}

La eficiencia de la combustión se determinó con el uso del analizador de gases BACHARACH PCA 3, el cual arrojó en la primera medición una eficiencia de la combustión de 87,6 \% y la segunda de $87,2 \%$, registrados en la tabla 11 con lo que se concluye que en los dos casos es alta, los porcentajes de gases de la combustión de gasoil en su mayoría están dentro del rango de valores típicos, según (ONAGAS, 2017) y (TESTO). 
Tabla 11: Eficiencia de la combustión

\begin{tabular}{|c|c|c|c|c|}
\hline $\begin{array}{l}\text { GASES DE LA } \\
\text { COMBUSTIÓN }\end{array}$ & TESTO & ONAGAS & $\begin{array}{l}\text { MEDICIÓN } \\
1\end{array}$ & $\begin{array}{l}\text { MEDICIÓN } \\
2\end{array}$ \\
\hline Oxígeno (\%) & $2-8$ & $2-5$ & 0,055 & 0,055 \\
\hline Monóxido de Carbono (ppm) & $40-150$ & $80-150$ & 28 & 25 \\
\hline Dióxido de Carbono (\%) & $11-14$ & $12,5-14$ & 0,115 & 0,115 \\
\hline Óxido de Nitrógeno (ppm) & $20-100$ & $50-100$ & 16 & 17 \\
\hline $\begin{array}{l}\text { Temperatura del gas de de } \\
\text { chimenea }\left({ }^{\circ} \mathrm{C}\right)\end{array}$ & a de la & $90-150$ & 155 & 168 \\
\hline Temperatura de agua $\left({ }^{\circ} \mathrm{C}\right)$ & & & 85 & 100 \\
\hline Eficiencia de la combustión (\% & & & 87,60 & 87,20 \\
\hline
\end{tabular}

Fuente: Autor, Ecuador, 2018

Los valores altos de eficiencia en la combustión se le atribuyen a que el quemador utilizado en la operación es nuevo y se utiliza el máximo flujo de combustible, al estar los valores dentro del rango se concluye que ambientalmente el impacto no es considerable, sin embargo al utilizar la caldera con un combustible fósil se genera contaminación.

\section{Propuesta de Mejora.}

Un objetivo de este proyecto es el de sustituir parcialmente el uso de energías convencionales que queman combustibles fósiles, que causan gran contaminación ambiental y alto costo de funcionamiento por lo que en la propuesta de mejoras se analizará dos opciones que utilizan energías alternativas.

La Energía solar es una fuente de energía renovable que se obtiene al aprovechar la irradiación del sol y se la utiliza hoy en día para varias aplicaciones entre ellas sistemas de calentamiento de agua sanitaria, a través del uso de dispositivos llamados colectores solares.

Para el dimensionamiento del sistema de calentamiento solar se toma en cuenta la demanda de energía y el costo de la instalación.

\section{Recurso energético disponible}

La ubicación geográfica de Ecuador es un privilegio en lo que se refiere a recurso solar, la incidencia del sol es perpendicular lo que permite obtener valores altos de irradiación solar, los datos fueron proporcionados por la estación meteorológica de la ESPOCH los mismos que fueron tomados con un instrumento llamado Piranómetro quien entrega la irradiancia global en W/m $/ \mathrm{m}^{2}$ por hora, la figura 5 muestra en promedio las horas en las que la irradiancia es mayor desde enero a octubre de 2017.

En la ciudad de Riobamba, la radiación más alta se presenta desde las 9H00 hasta las 15H00, el valor más alto de radiación es de $798 \mathrm{~W} / \mathrm{m}^{2}$ que corresponde a las $12 \mathrm{H} 00$, de $0 \mathrm{H} 00$ a $5 \mathrm{H} 00$ y de 
$19 \mathrm{H} 00$ a 23H00 no hay valores registrados para la radiación, desde las $6 \mathrm{H} 00$ ya se detectan datos de presencia de sol en la ciudad de Riobamba.

Figura 5: Ciclo diario de radiación en Riobamba

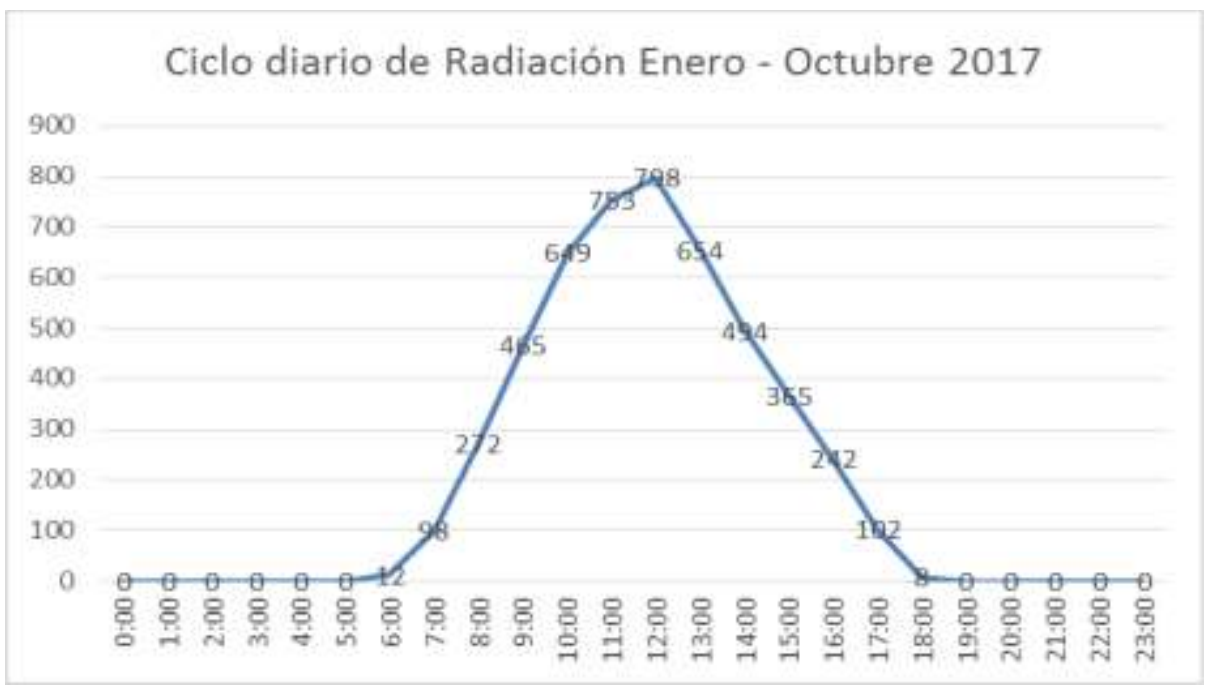

Fuente: Autor, Ecuador, 2018

Para determinar el número de colectores solares necesarios para un sistema de calentamiento de agua se calcula la radiación solar diaria, es decir se utiliza todos los valores obtenidos de radiación por hora y se suman, obteniéndose un valor al día de radiación, de esto se obtiene los valores promedios para cada mes y que se encuentran registrados en la tabla 12:

Tabla 12: Radiación solar en Riobamba

\begin{tabular}{ll}
\hline Mes & Radiación $\frac{W}{m^{2}}$ \\
\hline Enero & 5112,48 \\
Febrero & 4815,21 \\
Marzo & 4520,41 \\
Abril & 5236,08 \\
Mayo & 4987,65 \\
Junio & 5260,97 \\
Julio & 5239,81 \\
Agosto & 5546,72 \\
Septiembre & 5632,42 \\
Octubre & 5867,06 \\
Promedio & 5221,881 \\
\hline
\end{tabular}

Fuente: Autor, Ecuador, 2018 
En general los valores de radiación alcanzados en la ciudad de Riobamba son altos lo que nos permite trabajar con sistemas alternativos de calentamiento de agua.

\section{Selección de colectores solares}

Para determinar el número de colectores solares que satisfagan los requerimientos ideales de servicio se calcula las pérdidas de calor en el vaso de la piscina para $28^{\circ} \mathrm{C}$ de temperatura, que se la considera ideal para que los usuarios disfruten de este servicio, considerando que gran parte de los bañistas son niños.

Tabla 13: Resumen de pérdidas de calor en la piscina para $28^{\circ} \mathrm{C}$ de temperatura

\begin{tabular}{ll}
\hline Temperatura agua piscina & $28,00^{\circ} \mathrm{C}$ \\
\hline Hora & $11 \mathrm{H} 00$ \\
Número de nadadores & 28 \\
Número de espectadores & 6 \\
Pérdidas por evaporación & 39024,79 \\
Pérdidas por radiación & 14999,23 \\
Pérdidas por convección & 2059,87 \\
Pérdidas por renovación & 33213,93 \\
Pérdidas por conducción & 4283,60 \\
TOTAL (W) & 93581,43 \\
\hline
\end{tabular}

Fuente: Autor, Ecuador, 2018

Con el colector HC - 50 cuya área de transferencia de calor es de $4,65 \mathrm{~m}^{2}$ y la eficiencia es del $80 \%$, según la figura 6 :

Figura 6: Tipos de colectores solares

\begin{tabular}{|c|c|c|c|c|c|}
\hline Tipo de Colectores & & $\begin{array}{c}\text { HC.50 } \\
1.2 \times 3,85 \mathrm{~m} \\
4 \times 12.5^{\prime}\end{array}$ & $\begin{array}{c}\text { HC. }-40 \\
1,2 \times 3.23 \mathrm{~m} \\
4 \times 10.5\end{array}$ & $\begin{array}{c}\text { HC. } 38 \\
1,2 \times 2,92 \times n \\
4^{\prime} \times 10^{\prime}\end{array}$ & $\begin{array}{l}\mathrm{HC}-30 \\
\begin{array}{c}1,2 \times 2,31 \mathrm{~m} \\
4 \times 8^{\prime}\end{array}\end{array}$ \\
\hline Na. Cat do MEE & & 127112 & 127111 & 127110 & 127108 \\
\hline Large & $m$ & 3.85 & 3.23 & 2.82 & 2.31 \\
\hline Ancho. & m & 1.2 & 1.2 & 1.2 & 1.2 \\
\hline Superficie & $m^{*}$ & 4,65 & 3.,85 & 3.52 & 2.77 \\
\hline Peso "Seco" & $\mathrm{K}_{\mathrm{a}}$. & 10 & 8.5 & 8,2 & 6.8 \\
\hline Capocidod & LA. & 14.4 & 11.7 & 11.4 & 7.1 \\
\hline Pese "Hūmedo" & $\mathrm{Kg}$. & 24 & 20.5 & 19.6 & 15.8 \\
\hline No. de Espocoodoren & * & 12 & 10 & 9 & 7 \\
\hline Peaso de Área tiena & $\mathrm{Kg} . / \mathrm{m}^{2}$ & 5,16 & 5,3 & 5.3 & 5.7 \\
\hline Rec: Velooidod do Flujo & $\mathrm{Lt}$, / hr & 1200 & 950 & 900 & 120 \\
\hline
\end{tabular}

Fuente: (HELIOCOL, 2014)

Por lo expuesto se selecciona el colector $\mathrm{HC}$ - 50 cuya área de transferencia de calor es de 4,65 $\mathrm{m}^{2}$ y la eficiencia es del 80\%, se necesitan 93 paneles solares lo que representa un área de captación solar de $430 \mathrm{~m}^{2}$. 


\section{Sistema de calentamiento de agua con bombas de calor}

Otra alternativa a ser considerada para calentar el agua de la piscina son las bombas de calor que utilizan energía eléctrica para su funcionamiento, para determinar las características de estas se toma en cuenta las condiciones del sistema registradas en la tabla 4 y la temperatura de $28^{\circ} \mathrm{C}$ considerada como adecuada para satisfacer las necesidades de los bañistas se determinó que la potencia necesaria para que el sistema funcione eficientemente es de $312,44 \mathrm{~kW}$, con este dato en la figura 7 se selecciona el tipo de bomba de calor más adecuado.

Figura 7: Tipos de bomba de calor

\begin{tabular}{|c|c|c|c|c|c|c|c|c|c|c|}
\hline \multirow{2}{*}{ MODELO } & \multirow{2}{*}{ VOLTAGE } & \multirow{2}{*}{$\mathrm{HZ}$} & \multirow{2}{*}{$\begin{array}{c}\text { AMPERAJE } \\
\text { PROMEDIO } \\
\text { [AMP] } \\
\end{array}$} & \multirow{2}{*}{$\begin{array}{c}\begin{array}{c}\text { CAPACIDAD DE } \\
\text { CALENTAMIENTO }\end{array} \\
{[\mathrm{BTU} / \mathrm{H}]} \\
\end{array}$} & \multirow{2}{*}{ COP } & \multicolumn{2}{|c|}{ FLUJO [GPM] } & \multirow{2}{*}{$\begin{array}{l}\text { PESO } \\
\text { [LBS] }\end{array}$} & \multirow{2}{*}{ DIMENSIONES } & \multirow{2}{*}{$\begin{array}{c}\text { COMPRESOR } \\
\text { TIPO }\end{array}$} \\
\hline & & & & & & MIN & MAX & & & \\
\hline HE 125 RA & $208-230 / 1 \mathrm{~F}$ & 60 & 32 & 125.000 & 6.3 & & & & & \\
\hline HE 125 RB & $209-230 / 3 F$ & 60 & 23 & 125.000 & 6.3 & 20 & 70 & 269 & $31^{\prime \prime}$ x 31"x 43" & Scroll \\
\hline HE 150 RA & $210-230 / 1 \mathrm{~F}$ & 60 & 40 & 136.000 & 6 & & & & & \\
\hline HE $1 5 0 \longdiv { R B }$ & $211-230 / 3 F$ & 60 & 28 & 140.000 & 6 & & & & & \\
\hline
\end{tabular}

Fuente: (GULFSTREAM, 2014)

Se necesita 8 bombas de calor HE 150-RB.

\section{Comparación entre las alternativas de solución}

A continuación se realiza una comparación que ayudará a determinar cuál es la propuesta más factible, en la tabla 14 se encuentran las principales características de cada sistema.

Tabla 14: Comparación entre alternativas de solución (tabla en Excel no gráfico)

\begin{tabular}{|l|c|c|}
\hline SISTEMAS DE CALENTAMIENTO & PANELES SOLARES & BOMBA DE CALOR \\
\hline Costo de equipos e instalación & $\mathbf{4 4 4 . 8 1 5 , 6 5}$ & $\mathbf{\$}$ \\
\hline Mantenimiento & Muy poco & $\mathbf{4 6 . 3 6 8 , 0 0}$ \\
\hline Consumo de combustible & Sí & Nocuente \\
\hline Consumo de energía eléctrica & No & SÍ \\
\hline Tiempo de vida útil & 25 años & $20-25$ años \\
\hline
\end{tabular}

Fuente: Autor, Ecuador, 2018 
Si se compara el costo de los dos sistemas la alternativa del uso de las bombas de calor es la más costosa, el mantenimiento para este sistema es preventivo y se lo hace con frecuencia ya que se necesita limpiar los filtros y revisar las instalaciones hidráulicas, su funcionamiento depende exclusivamente del uso de energía eléctrica, no hay consumo de diesel ya que es un sistema independiente lo que representaría una eliminación total de gases contaminantes.

Mientras que el sistema que utiliza colectores solares necesita una inversión menor, en este caso se tendría un sistema combinado que utilice diesel por algunas horas y luego mantenga la temperatura del agua de la piscina con el uso de colectores solares, se eliminaría parcialmente la contaminación ambiental producida por el uso de la caldera, necesitaría muy poco mantenimiento y el tiempo de vida útil relativamente es el mismo para los dos sistemas.

\section{Conclusión}

- Se desarrolló una auditoria energética preliminar en la que se identificó que el actual sistema de calentamiento de agua es deficiente, la temperatura del agua de la piscina asciende hasta $26,6^{\circ} \mathrm{C}$, el rendimiento de la caldera oscila entre $53,4 \%$ y $58,9 \%$.

- Se determinó que cada día se necesitan $93,581 \mathrm{~kW}$ como el sistema funciona 13 horas la energía consumida es de $1216,55 \mathrm{~kW}$-h para alcanzar la temperatura en el agua de la piscina de $28^{\circ} \mathrm{C}$, considerada como adecuada para niños desde los 5 años de edad que entrenan en el complejo de natación Carlos Costales.

- Para calentar el agua de la piscina se analizaron dos sistemas con alternativas renovables, la primera consiste en un sistema híbrido que utiliza diesel durante 4 horas para elevar la temperatura del agua de $24,4^{\circ} \mathrm{C}$ a $28^{\circ} \mathrm{C}$ y luego para mantener estable la temperatura se utilizarán 93 paneles solares; la segunda alternativa es el uso de 8 bombas de calor cuyo funcionamiento dependerá exclusivamente del uso de energía eléctrica este consiste en un sistema independiente en el que no es necesario utilizar diesel.

\section{Referencias Bibliograficas}

Linkohr, R. (2006). LA POLITICA ENERGETICA LATINOAMERICANA ENTRE EL ESTADO Y EL MERCADO. Nueva Sociedad, 90-103.

Ciatesa. (28 de 11 de 2005). www.ciatesa.es. Recuperado el 10 de 06 de 2017 cosasdepiscina.wordpress.com. (12 de 11 de 2014). Recuperado el 18 de 02 de 2018 globalpetrolprices.com. (05 de 02 de 2018). Recuperado el 12 de 02 de 2018 BACHARACH. (03 de 08 de 2014). www.MyBacharach.com. Recuperado el 27 de 06 de 2017 Muños, M. (2012). Análisis comparativo de gases de combustión de calderas bagaceras. ONAGAS. (15 de Enero de 2017). http://onagas.es/analisis-de-combustion/. Recuperado el 27 de Diciembre de 2017

TESTO. (s.f.). www.testo.es. Recuperado el 27 de Diciembre de 2017

GULFSTREAM. (2014). BOMBAS DE CALOR. BOMBAS DE CALOR, 2.

HELIOCOL. (02 de 02 de 2014). CALENTADORES SOLARES. HELIOCOL, 2. 
Medioambiente, M. d. (11 de Agosto de 2010). Acuerdo $N^{\circ}$ 131. Obtenido de http://www.ambiente.gob.ec/wp-content/uploads/downloads/2014/04/ACUERDO-

MINISTERIAL-131.pdf

\section{PARA CITAR EL ARTÍCULO INDEXADO.}

Moreno Pinduisaca, L., Palacios Almache, J., Buenaño Moyano, L., \& Barrera Cárdenas, O. (2019). Diagnóstico energético del sistema de calentamiento de agua en la piscina del Complejo de Natación Carlos Costales de la ciudad de Riobamba. Propuesta de alternativas de solución. Ciencia Digital, 3(2.6), 370-387. https://doi.org/10.33262/cienciadigital.v3i2.6.572

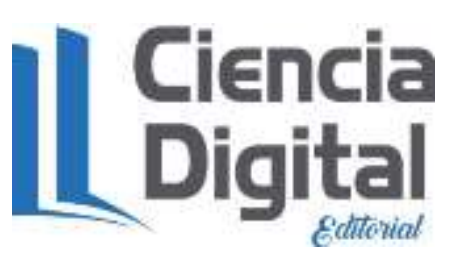

El artículo que se publica es de exclusiva responsabilidad de los autores y no necesariamente reflejan el pensamiento de la Revista Ciencia Digital.

El artículo queda en propiedad de la revista y, por tanto, su publicación parcial y/o total en otro medio tiene que ser autorizado por el director de la Revista Ciencia Digital.
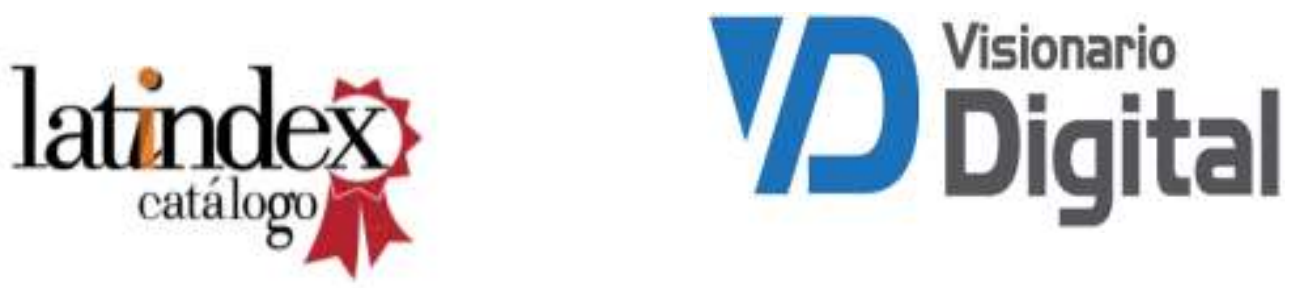